\title{
Intervenciones en la administración de medicamentos de alto riesgo
}

\author{
Interventions in the administration of high-risk drugs \\ Dafne Liseth Romero Gutiérrez,* Alma Lidia Almiray Soto, ${ }^{\ddagger}$ \\ Eduardo Ensaldo Carrasco§
}

\begin{abstract}
RESUMEN
Introducción: Los errores de medicación representan una de las prioridades de seguridad del paciente. Desde el año 2017, la Organización Mundial de la Salud declaró a la medicación segura como el tercer reto global. Una de las áreas estratégicas para prevenir los errores de medicación son las buenas prácticas en la administración de medicamentos de alto riesgo. Para lograrlo, es crucial el involucramiento del personal de enfermería, dado que representan un recurso humano valioso para el sistema de salud y el procuramiento de la seguridad del paciente. En este contexto, los medicamentos de alto riesgo son un tipo de fármacos con alto potencial de ocasionar daños graves o, incluso, mortales. Por lo tanto, en este artículo se presenta un listado de recomendaciones para la administración segura de medicamentos de alto riesgo por el personal de enfermería.
\end{abstract}

Palabras clave: Seguridad del paciente, enfermería, preparaciones farmacéuticas, errores de medicación.

\begin{abstract}
Introduction: Medication error represent a patient safety priority. Since 2017, the World Health Organization (WHO) declared medication safety as the third global challenge. A key area for the prevention of medication errors are good practices for administering high-risk medications. To achieve this, is crucial that nursing professionals are involved as they represent a valuable human resource for the health system and ensuring patient safety. In this context, high-risk medications possess a high risk to cause severe harm o even death. Therefore, this article presents a list of recommendations for the safe administration of high-risk medications.
\end{abstract}

Keywords: Patient safety, nursing, pharmaceutical preparations, medication errors.

\section{INTRODUCCIÓN}

La medicación segura es una de las prioridades globales en materia de la calidad y la seguridad del paciente de los sistemas de salud.' Los errores de medicación representan una de las prioridades en el mundo. ${ }^{2}$ Se estima que, en los Estados Unidos de América, los errores de medicación ocasionan al menos una muerte diaria y daños en aproximadamente 1.3 millones de personas al año. ${ }^{3}$ El costo global asociado a los errores de medicación se ha estimado en 42 billones de dólares anuales. ${ }^{4}$ En el año 2017, la Organización Mundial de la Salud (OMS) anunció el tercer reto global para la seguridad del paciente para reducir, en un periodo de cinco años, el 50\% de los daños graves y evitables relacionados con la medicación. ${ }^{5}$ En el mismo año, entre
* Programa de Servicio Social en Investigación (SSICONAMED). Facultad de Estudios Superiores Iztacala. UNAM.

¥ Unidad de Investigación en Epidemiología Clínica. Hospital Infantil de México «Federico Gómez».

${ }^{\S}$ Comisión Nacional de Arbitraje Médico. México.

\section{Correspondencia:}

DLRG, dafne.romero.g@ gmail.com

\section{Conflicto de intereses:}

Los autores declaran que no tienen.

Citar como: Romero GDL, Almiray SAL, Ensaldo CE. Intervenciones en la administración de medicamentos de alto riesgo. Rev CONAMED. 2020; 25(2): 95-97. doi: $10.35366 / 94393$

Financiamiento: No recibió ningún tipo de apoyo económico o financiamiento del sector público, comercial, o sin ánimo de lucro.

Recibido: 22/06/2020 Aceptado: 23/06/2020 
las estrategias de México para mejorar la calidad de los servicios de salud, se emitieron ocho acciones esenciales para la seguridad del paciente. ${ }^{6}$ La tercera acción trata sobre la seguridad en el proceso de medicación, la cual busca «Fortalecer las acciones relacionadas con el almacenamiento, la prescripción, transcripción, dispensación y administración de medicamentos, para prevenir errores que puedan dañar a los pacientes». ${ }^{7}$ En este contexto, los medicamentos de alto riesgo son un tipo de fármacos con alto potencial de ocasionar daños graves o incluso mortales, ${ }^{8}$ incluyen medicamentos citotóxicos, insulinas, anticoagulantes y electrolitos concentrados. ${ }^{9}$ En respuesta a ese objetivo, se presentan recomendaciones generales para la administración segura de medicamento de alto riesgo.

\section{RECOMENDACIONES}

1. Los medicamentos de alto riesgo en áreas de atención al paciente se deben retirar si no se justifica desde el punto de vista clínico su existencia o pertenencia.

2. El almacenamiento de los medicamentos de alto riesgo, tanto en la farmacia como en los Centros de Distribución de medicamentos en Área de Hospitalización (CENDIS), deben de estar en contenedores en la parte superior y central del casillero, en un lugar seguro y diferente del resto de los medicamentos, con las condiciones ambientales que el laboratorio farmacéutico indique.

3. Etiquetar los contenedores de los medicamentos que son considerados de alto riesgo, con la leyenda «Medicamento de alto riesgo». Los medicamentos de alto riesgo deberán estar etiquetados de manera individual con un círculo rojo.

4. En la prescripción: los médicos deben realizar la prescripción médica con letra clara, legible sin abreviaturas, sin enmendaduras ni tachaduras. Se prohíbe el uso de la abreviatura «U» para indicar unidades.

5. Los medicamentos con aspecto o nombre parecido "LASA (Look alike, sound alike)» se deberán rotular con letras mayúsculas las letras diferentes, ejemplo: DIGOxina DORIxina. En el proceso de almacenamiento y prescripción de los medicamentos LASA, colocar alertas visuales dando prioridad al proceso de prescripción.

6. En aquellos medicamentos donde el envase es similar entre las distintas presentaciones o claves, se deben diferenciar para evitar confusión en el personal de enfermería, especialmente en el almacenaje, la ubicación y el etiquetado.

7. Transcripción del medicamento: el personal de enfermería transcribirá en la hoja de enfermería los medicamentos y las indicaciones médicas sin modificar la prescripción original. En caso de duda debe aclararlas con el médico tratante. Solicitará el medicamento a la farmacia de la unidad de salud.

8. Dispensación del medicamento: el personal de la farmacia verificará la solicitud del medicamento. En caso de dudas, no debe entregar el medicamento y se pondrá en contacto con el médico que prescribe para realizar la aclaración. Proporcionará la información completa sobre su manejo a quien lo recibe.

9. En la recepción y almacenamiento de los medicamentos: las enfermeras rotularán el medicamento con el nombre completo del paciente y sus datos de identificación (fecha de nacimiento por día, mes y año) en su caso, adicionalmente podrán agregarse otros determinados en la normativa de la institución, ejemplo: el número de registro.

10. La preparación y administración del medicamento lo ejecutará el mismo profesional de enfermería que lo recibió. No precargar la medicación.

11. No se administrarán medicamentos que carezcan de etiquetas o cuando éstas no sean legibles.

12. La enfermera o, en su caso, el médico, deberán preguntar al paciente si es alérgico y verificará los datos en el identificador personal y la ficha de identificación del paciente, antes de la administración de cualquier medicamento.

13. Utilizar antes, durante y después de la transcripción, preparación y administración de medicamentos las indicaciones correctas de la medicación. El personal de enfermería que administre los medicamentos deberá verificar que:

a. El paciente sea el correcto, preguntando al paciente su nombre completo y fecha de 
nacimiento y verificar que éstos coincidan con la nota de clínica y de enfermería.

b. El medicamento sea el correcto, identificando siempre el medicamento al retirarlo de la zona de almacenamiento y verificar el nombre del medicamento con las indicaciones del expediente clínico del paciente y con la hoja de enfermería.

c. La dosis sea la correcta, comprobando en los registros del expediente y en la hoja de enfermería la dosis prescrita.

d. La vía sea la correcta, confirmando la vía de administración del medicamento indicada en la etiqueta, expediente clínico y la hoja de enfermería.

e. El horario sea el correcto, confirmando que en el expediente clínico y en la hoja de enfermería se describan los intervalos indicados para la administración del medicamento.

f. El registro de los medicamentos sea el correcto. Inmediatamente después de la administración, registrar en el expediente clínico y en la hoja de enfermería el nombre del fármaco, fecha, hora, dosis y vía de administración, así como el nombre de quien la administró.

14. Los medicamentos prescritos para infusión intravenosa deben ser diluidos y no mezclados.

15. Los medicamentos cuya administración se suspenda por indicación médica, deben ser regresados a la farmacia.

16. En caso de que se omita administrar un medicamento, debe registrarse en el expediente clínico y en la hoja de enfermería, señalando claramente las causas por las que no se administró.

17. La administración de los medicamentos de alto riesgo requiere de una doble verificación para su administración. Esto se realiza por dos personas con las competencias idóneas, durante los procesos de preparación (primera verificación) y en la administración (segunda verificación). Deben firmar en los registros clínicos del expediente las personas que realizaron este procedimiento. La doble verificación se realiza en las áreas establecidas por la institución de salud para preparación y ministración de medicamentos de alto riesgo para prevenir errores de medicación.

\section{Bibliografía}

1. OMS. Seguridad del paciente. Medidas mundiales en materia de seguridad del paciente. $72^{\mathrm{a}}$ Asamblea Mundial de la Salud. Organización Mundial de la Salud; 2019.

2. Briefing Note Safety of medicines - adverse drug reactions Key facts. [Cited 2020 May 27]; 1-3. Available from: https:// www.who.int/medicines/regulation/medicines-safety/M_ SBN_Jun78.pdf?ua=1.

3. La OMS lanza una iniciativa mundial para reducir a la mitad los errores relacionados con la medicación en cinco años [Internet]. [Cited 2020 May 20]. Available from: https://uww. who.int/es/news-room/detail/29-03-2017-who-launchesglobal-effort-to-halve-medication-related-errors-in-5-years.

4. Donaldson LJ, Kelley ET, Dhingra-Kumar N, Kieny MP, Sheikh A. Medication without harm: WHO's third global patient safety challenge. Lancet. 2017; 389 (10080): 1680-1687.

5. Sheikh A, Dhingra-Kumar N, Kelley E, Kieny MP, Donaldson LJ. The third global patient safety challenge: tackling medication-related harm. Bull World Health Organ. 2017; 95 (8): 546-546A.

6. Acuerdo por el que se declara la obligatoriedad de la implementación, para todos los integrantes del Sistema Nacional de Salud, del documento denominado Acciones Esenciales para la Seguridad del Paciente [Internet]. [Cited 2020 Jan 6]. Available from: http://webcache.googleusercontent.com/ search?q=cache:38WQxr5daDgJ:dof.gob.mx/nota_to_ doc.php\%253Fcodnota\%253D5496728+\&cd=1\&hl=es419\&ct=clnk\&gl=mx

7. De Salubridad General C. Sistema Nacional de Certificación de Establecimientos de Atención Médica. Las 2 acciones esenciales para la seguridad del paciente dentro del modelo de seguridad para el paciente del CSG. Edición 2017. Ciudad de México: Consejo de Salubridad General; 2017.

8. Cohen MR, Smetzer JL, Tuohy NR, Kilo CM. High-alert medications: safeguarding against errors. Medicat Errors 2nd ed Washingt Am Pharm Assoc. 2007, p. 317-411.

9. Intervenciones de Enfermería para la Seguridad en la Administración de Medicamentos de Alto Riesgo: Guía de Práctica Clínica. México, CENETEC; 2014 [28 de Diciembre 2019]. Disponible en: http://www.cenetec-difusion.com/ CMGPC/IMSS-712-14/ER.pdf. 